\title{
Septische Gonarthritis und ausgedehnter Wadenabszess durch Streptococcus intermedius bei einem 4-jährigen Jungen
}

Streptococcus intermedius bildet mit Streptococcus anginosus und Streptococcus constellatus die Streptococcus-anginosusGruppe (SAG) [1], die zu den Viridans-Streptokokken gehört [2]. Sie sind Kommensalen der Oropharyngeal-, Gastrointestinal- und Urogenitalflora [3]. Die SAG ist potenziell pyogen, wobei Streptococcus intermedius der pathogenste Keim der Gruppe ist. Er kann vor allem Abszess-Formationen in Hirn und Leber verursachen [2,4] und selten auch zu Endokarditis und Milzabszessen führen [4, 5]. Typische Streptokokken-Virulenzfaktoren wie Hyaluronidase und Neuraminidase, die die Ausbreitung der Infektion begünstigen [2], wurden in Genomstudien identifiziert, funktionelle Studien der molekularen Basis fehlen jedoch [3]. Das vom Bakterium produzierte Cytolysin, das zur Streptolysin-O-Gen-Familie gehört, ist vermutlich maßgeblich an der Abszessbildung beteiligt [2]. Als Erreger einer osteoartikulären Infektion werden sie in der Literatur in der Regel nicht erwähnt.

\section{Fallbericht}

Wir berichten über einen 4-jährigen, immunkompetenten Jungen, der mit Laufverweigerung, Schwellung, Rötung, Überwärmung und Schmerzen des linken Kniegelenks seit 7 Tagen vorgestellt wurde.

Der Junge war bereits zwei Tage stationär in einem auswärtigen Krankenhaus, hier wurde eine Fraktur röntgenologisch ausgeschlossen, die Entlassung erfolgte gegen ärztlichen Rat. Bei nun auch fieberndem Kind erfolgte die Vorstellung 3 Tage später in unserer Kinderklinik.

Laborchemisch zeigten sich bei dem jetzt deutlich beeinträchtigten Kind stark erhöhte Entzündungszeichen (CrP 225 mg/l, Leukozyten $20 \mathrm{G} /$, Blutsenkungsgeschwindigkeit $70 \mathrm{~mm} / \mathrm{h}$ ) im Blut. In der Kniegelenkspunktion am Aufnahmetag wurden $45 \mathrm{ml}$ rahmiger Eiter mit $260000 / \mu$ l Leukozyten, davon $59 \%$ Granulozyten, aspiriert ( Abb. 1).

Es wurde umgehend eine kalkulierte, intravenöse Therapie mit Cefuroxim (150 mg/kg KG in 3 Dosen) begonnen sowie regelmäßig

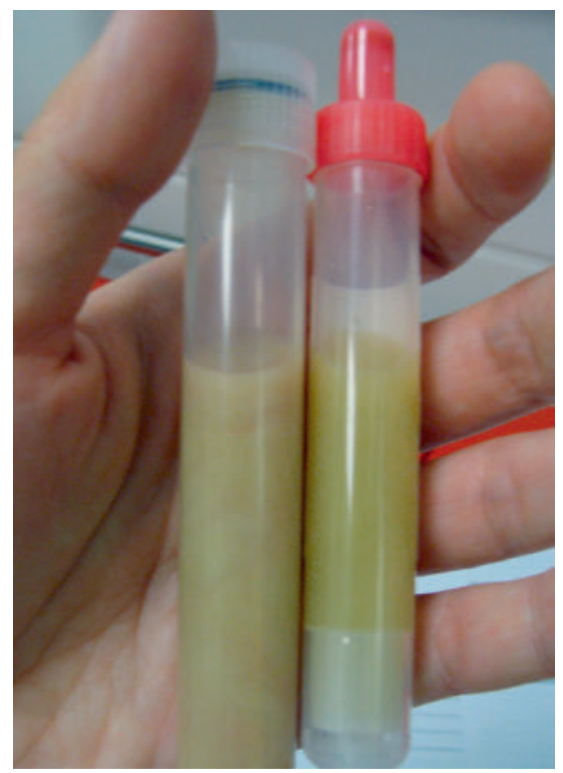

Abb. 1 Krankenhaustag 1. Deutlich eitrig wirkendes Punktat aus dem Knie.

Ibuprofen p. o. und über 4 Tage Dexamethason i.v. $(0,6 \mathrm{mg} / \mathrm{kg} \mathrm{KG}$ in 4 Dosen) verabreicht. In den aeroben Kulturen des Gelenkpunktats konnte mittels PCR Streptococcus intermedius nachgewiesen werden. Die im Grampräparat hohe Keimzahl schloss eine Verunreinigung der Kulturen aus. Anaerobe Kulturen sowie aerobe und anaerobe Blutkulturen blieben steril.

Trotz deutlicher Besserung der Klinik und anhaltender Fieberfreiheit an Tag 2, verweigerte der Junge das Laufen und die volle Gelenksextension.

Trotz intravenöser antibiotischer Therapie hatte sich in wenigen Tagen eine große Abszess-Formation der Flexorenloge des linken Unterschenkels ohne Nachweis einer Kniegelenksverbindung in der Bildgebung gebildet ( $\triangleright$ Abb. $\mathbf{2}$ bis $\$ Abb. $\mathbf{6}$ ). Die septierte Abszess-Formation wurde chirurgisch entlastet und mit einer Drainage versorgt. Molekularbiologisch konnte auch hier noch Streptococcus intermedius isoliert werden. Die antibiotische Therapie wurde auf Clindamycin (40 mg/kg KG in 3 Dosen) und Penicillin (375000 IE/kg KG in 4 Dosen) intravenös umgestellt. In Kontrollen durch Abdomen-Sonografie und Echokardiografie konnten keine weiteren Abszesse darge-

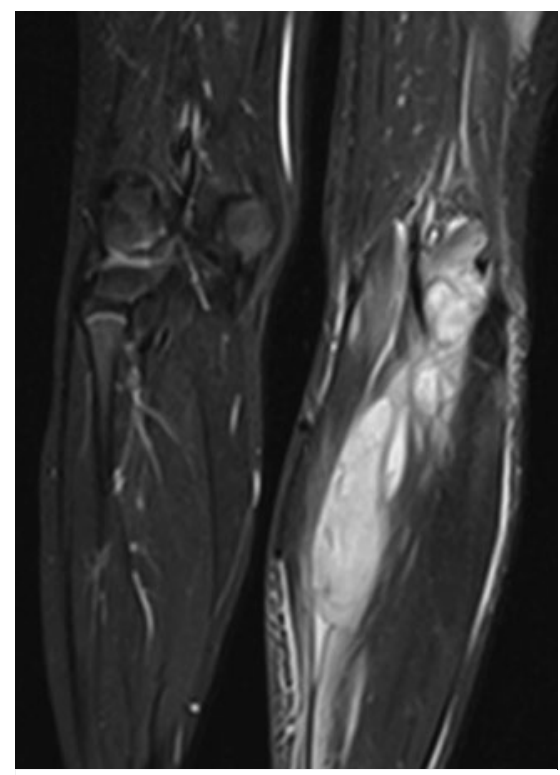

Abb. 2 Krankenhaustag 7. MRT T2STIR-Wichtung, coronar. Ausgedehnter, krankhafter Prozess linke Wade.

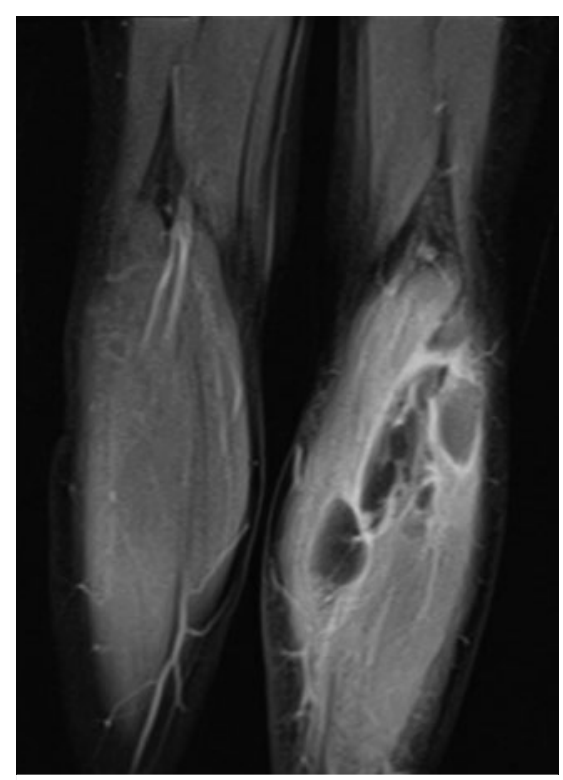

Abb. 3 Krankenhaustag 7.MRT T1 FS post KM, coronar. Krankhafter Prozess mit $11,5 \mathrm{~cm}$ Längsausdehnung linke Wade.

stellt werden, auch eine Bakteriämie konnte zu keiner Zeit nachgewiesen werden.

Nach insgesamt 21 Tagen intravenöser Therapie konnte die Antibiose oralisiert und der Junge nach Hause entlassen werden. Die Therapie wurde über weitere 2 Wo- 


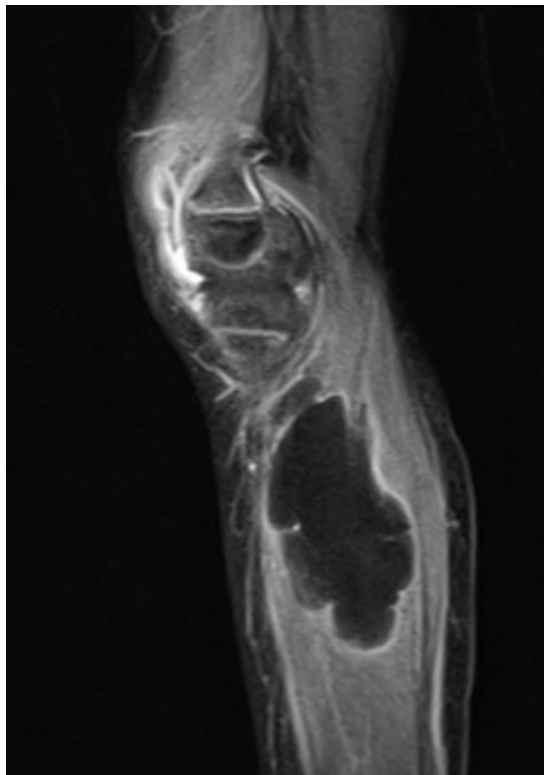

- Abb. 4 Krankenhaustag 7. MRT T1 FS post KM, sagittal. Krankhafter Prozess mit $8 \mathrm{~cm}$ Längsausdehnung linke Wade.

chen fortgeführt. Bei anhaltender Spitzfußstellung in der nachstationären Kontrolle bei normalisierten Entzündungswerten (CRP $0,3 \mathrm{mg} / \mathrm{l}$, BSG $8 \mathrm{~mm} / \mathrm{h}$ ) und anhaltender Schwellung des Knies wurde 1 Woche nach Therapieende eine intraartikuläre Steroidinjektion (10 mg Triamcinolonhexacetonid) durchgeführt. Nach intensiver Physiotherapie ist der Junge nun anhaltend vollkommen beschwerdefrei. Klinisch hatte der Junge zuvor keine häufigen oder ungewöhnlichen Infektionen durchgemacht und alle Impfungen einschließlich Lebendimpfungen gut vertragen.

Bei einer basalen Diagnostik hinsichtlich Immundefekt 20 Wochen später fanden sich keine wegweisenden Befunde. Untersucht wurden lgG inklusive Subklassen, IgM und IgA, Lymphozytenoberflächenmarker, Impfantiköper gegen Polio, Diphtherietoxin, Tetanustoxin und Pneumokokkenantigene sowie Isoagglutinine und C3, C4.

\section{Diskussion}

Streptococcus intermedius verursachte eine septische Gonarthritis bei einem gesunden, immunkompetenten 4-Jährigen Jungen. Trotz intravenöser Monotherapie mit Cefuroxim kam es zur Ausbildung einer großen Abszess-Formation der Wadenmuskulatur. Eine Besserung der Klinik trat

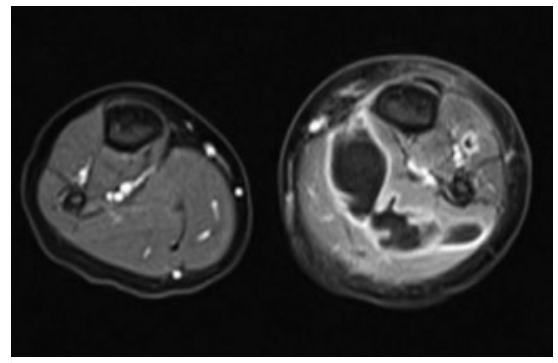

- Abb. 5 Krankenhaustag 7. MRT T1 FS post KM, axial. Proximal septierter Flüssigkeitsverhalt mit starkem Randenhancement, ca. $5,5 \times 2,5 \mathrm{~cm}$ groß linke Wade.

erst nach chirurgischer Entlastung und Drainage des Abszesses in Kombination mit Clindamycin und Penicillin i. v. über 14 Tage ein. Osteoartikuläre Infektionen können die Extremität oder sogar das Leben der Patienten bedrohen [7]. In der Regel lässt sich Staphylococcus aureus oder bei Kindern unter 4 Jahren häufig auch Kingella kingae als Erreger aus Punktat, seltener aus Blutkulturen isolieren [7]. Das Fehlen einer Bakteriämie, die als Zeichen eines schweren klinischen Verlaufs zu werten wäre, und die Abwesenheit von Fieber [2] in dem hier vorgestellten Fall, spiegelten das Ausmaß des Abszesses nicht wider.

So zeigte auch eine Kohorten-Studie (23 Fälle), bei der Blutkulturen bei Kindern mit Streptococcus-intermedius-Infektion untersucht wurden, nur zweimal einen positiven Nachweis einer Bakteriämie [3]. Wird ein ungewöhnlicher Keim als Ursache einer osteoartikulären Infektion vermutet, sollte dies mit den Labormedizinern kommuniziert werden, um einer Bewertung der Ergebnisse z. B. als Kontamination entgegen zu wirken, oder gegebenfalls vom Standard abweichende Prozeduren, wie eine längere Inkubationszeit, anzuwenden [7]. In weiteren Studien konnte gezeigt werden, dass in 33-55\% der Fälle osteoartikulärer Infektionen kein Bakterium identifiziert werden konnte [7]. Die hohe Keimbelastung des eingesendeten Materials und eine Bewertung durch ein 2. Labor (Prof. Dr. R. Bialek, Geesthacht) konnte in diesem Fall eine Kontamination der Proben durch Streptococcus intermedius ausschließen.

Weiterhin wurde dem hier vorgestellten Patienten zusätzlich Dexamethason zur Beschleunigung der Heilung und Prävention

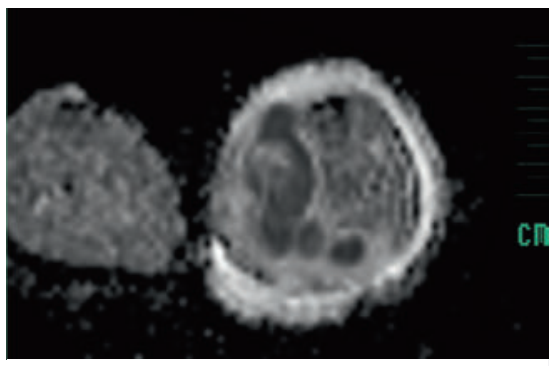

- Abb. 6 Krankenhaustag 7. MRT ADC map, axial. Nachweis einer Diffusionsstörung im krankhafter Prozess, in Zusammenschau der MRT-Bildgebung: Abszess im M. gastrocnemius links.

von Spätschäden verabreicht. Eine Kohorten-Studie zeigte, dass zusätzliche adjuvante Gaben von Dexamethason bei septischer Arthritis der antibiotischen Monotherapie in Bezug auf klinischem Verlauf und Krankheitsdauer überlegen ist [4]. Studien bei septischer Arthritis durch Streptococcus intermedius und Dexamethason-Therapie liegen nicht vor. Ob die systemische Verabreichung eines steroidhaltigen Präparats zu einer weiteren Schwächung der Immunabwehr geführt hat und damit die Abszessausbreitung weiter begünstigte, bleibt offen, erscheint uns aber angesichts der genannten bekannten Pathogenität des Keimes unwahrscheinlich. Letztlich war in diesem Fall die antibiotische Monotherapie nicht ausreichend wirksam, um die Bildung eines neuen Abszesses zu unterdrücken. Die chirurgische Entlastung war für die Therapie unseres Patienten entscheidend. In der Literatur wird nur in $12 \%$ der Fälle eine chirurgische Intervention (üblicherweise wiederholte Punktion und Arthroskopie/-tomie zur Gelenkspülung und Débridement) bei septischer Arthritis als notwendig beschrieben [7]. Dies unterstreicht erneut die Schwere der Infektion des hier vorgestellten Patienten.

Die Pathogenität der SAG wird oft unterschätzt, weil die Speziesidentifikation schwierig ist und die SAG Kommensalen der Mukosa sein können und daher oft als Kontaminanten fehlgedeutet werden können [3]. Streptococcus intermedius ist aber ein höchst virulenter humanpathogener Erreger [1, 2]. Es können junge und immunkompetente Patienten von Abszessbildung betroffen sein und es sollte bei Nachweis von Streptococcus intermedius gegebenenfalls auch nach sekundären Abszessen gesucht werden. 


\section{ZUSAMMENFASSUNG}

Berichtet wird von einem 4-Jährigen, bislang gesunden Jungen, mit septischer Gonarthritis bedingt durch Streptococcus intermedius. Trotz resistogrammgerechter intravenöser antibiotischer Therapie mit Cefuroxim unmittelbar nach diagnostischer Kniegelenkspunktion, entwickelte sich innerhalb weniger Tage eine große Abszess-Formation der Flexorenloge des Unterschenkels der gleichen Extremität, ohne Nachweis einer Kniegelenksverbindung in der Bildgebung. Molekularbiologisch konnte auch hier Streptococcus intermedius isoliert werden. Eine Besserung der Klinik trat erst nach chirurgischer Entlastung und Drainage des Abszesses in Kombination mit Clindamycin und Penicillin i. v. über 14 Tage auf. Streptococcus intermedius kann ein hoch virulenter humanpathogener Erreger sein, der vor allem ZNS- und abdominelle Abszess-Formationen verursachen kann. Der vorgestellte Fall zeigt, dass der Erreger daneben auch eine septische Arthritis und einen Weichteilabszess bedingen kann.

\section{Interessenkonflikt}

Die Autoren geben an, dass kein Interessenkonflikt besteht.

\section{Einhaltung ethischer Richtlinien}

Der korrespondierende Autor bestätigt, dass die Eltern des Patienten ihr Einverständnis gegeben haben, den Fall zu publizieren.

\section{Danksagung}

Die Autoren danken dem Kinderradiologen K. Jablonka herzlich für die Beratung bei der Auswahl sowie für die Befundung der MRT-Bilder.

\section{Autorinnen/Autoren}

\section{Zachries, P. Kaiser-Labusch, F. Weller-Heinemann, H.I. Huppertz \\ Prof.-Hess-Kinderklinik Bremen, Gesundheit Nord gGmbH}

\section{Korrespondenzadresse}

\section{F. Weller-Heinemann}

OA Kinder- und Jugendrheumatologie Prof. Hess Kinderklink Bremen, Gesundheit Nord gGmbH Friedrich Karl Straße, 28177 Bremen frank.weller@klinikum-bremen-mitte.de

\section{Literatur}

[1] Asam DS. Molecular pathogenicity of Streptococcus anginosus. Mol Oral Microbiol 2014; 29: 145-155

[2] Dodwell ER. Osteomyelitis and septic arthritis in children: current concepts. www. co-pediatrics.com. Lippincott Williams and Wilkins, 2013; 25(1)

[3] Faden $\mathrm{H}$. Infections associated with Streptococcus intermedius in children. The pediatric Infectious Disease Journal 2016; 35(9): 1047

[4] Itay Fogel JA-O. Dexamethasone Therapy for Septic Arthritis in Children. American Academy of Pediatrics, 2015

[5] Maliyil JC. Splenic abscess and multiple brain abscesses caused by Streptococcus intermedius in a young and healthy man. Proc Bayl Univ Med Cent 2011; 24(3): 195-199

[6] Simone GR. Streptococcus anginosus group disseminated infection: case report and Iterature review. Le Infezioni in Medicina 2012; 3: 145-154

[7] Tran MP-M. Streptococcus intermedius causing infective endocarditis and abscesses: a report of three cases and review of the literature. BMC Infectious Diseases 2008; 8

Bibliografie

DOI https://doi.org/10.1055/a-0895-9087 arthritis + rheuma 2020; 40: 44-46 (c) Georg Thieme Verlag KG Stuttgart · New York ISSN 0176-5167 